\section{PWE-135 DOES AFP PREDICT SURVIVAL IN PATIENTS WITH HEPATOCELLULAR CARCINOMA (HCC)?}

${ }^{1}$ F Yousuf*, 'W Ding, ${ }^{1}$ PD Richardson, ${ }^{2}$ D Palmer, 'I Patanwala, ${ }^{1} \mathrm{TJ}$ Cross. ${ }^{1}$ Royal Liverpool and Broadgreen University Hospitals, UK; ${ }^{2}$ Academic Department of Medical Oncology, University of Liverpool, Liverpool, UK

\subsection{6/gutjnl-2014-307263.395}

Introduction AFP is a tumour marker that is elevated in some patients with HCC and has been recommended as a method of screening for HCC in at risk patients. The authors wished to assess the level of AFP at diagnosis to determine if this was related with survival.

Methods A single centre, retrospective cohort analysis was performed. Patients with HCC were identified from the department's database (2008-2014). The diagnosis of HCC was made on internationally agreed criteria. Screening for HCC was based on 6-monthly liver ultrasound and serum measurement of AFP. The point of diagnosis was taken as the date when the diagnosis was confirmed radiological or histologically. The severity of patient's disease was scored using the Barcelona Liver Cancer Clinic (BCLC) classification. The serum AFP was measured at diagnosis. The end-point for the study was patient death.

Results 180 patients were identified in our cohort. The median age was 68 years (58-75), the number of males was $142(79 \%)$, and aetiology was as follows: ALD (50, 39\%), NASH (17, 13.3\%), HCV (22, 17.2\%), HBV (10, 7.8\%), haemochromatosis $(10,7.8 \%)$, others $19(14.8 \%)$. The stage of BCLC at presentation was: 0-18 (10\%), A - 43 (24\%), B 30 (16.7\%), C 19 $(10.6 \%)$, and D 25 (13.3\%). Survival at 1 and 5 years from diagnosis was $69 \%$ and $46 \%$ respectively. AFP was available in 93 cases. AFP was elevated $(>10)$ in 37 cases $(40 \%)$. On univariate analysis the following variables were associated with a poor outcome at 1 year (AFP $\mathrm{p}=0.001$, tumour diameter 0.06 ) and 5 years (AFP $\mathrm{p}<0.0001$ and tumour diameter 0.04 ). The median AFP in survivors at 5 years was 4(3-6), and in non-survivors was 97(24-673). An AFP >100 at presentation was associated with an increased risk of death at 1 (OR $4.5(2-10)$ and 5 years (OR 7.2(3-17) irrespective of treatment modality employed.

Conclusion AFP does have prognostic utility in patients with HCC. It is a poor screening tool as it is not elevated in the majority of patients with HCC. New biomarkers are needed to help earlier detection when the disease is at a potentially curative stage.

Disclosure of Interest None Declared.

\section{PWE-136 HEPATOCELLULAR CANCER DETECTED IN THE CIRRHOSIS SURVEILLANCE PROGRAMME HAVE BETTER OUTCOMES THAN THOSE DIAGNOSED SYMPTOMATICALLY}

F Clegg*, L Bailey, P Ramachandran, P Dundas, S English, L McLeman, B Vijayan, A Fraser, A Mukhopadhya. Gastroenterology, Aberdeen Royal Infirmary, Aberdeen, UK

\subsection{6/gutjnl-2014-307263.396}

Introduction Hepatocellular cancer (HCC) is a recognised complication of liver cirrhosis. ${ }^{1}$ The cause of liver cirrhosis is varied, but the prevalence of certain conditions like non-alcoholic fatty liver disease (NAFLD) is rising. ${ }^{2}$ This study aimed to identify the time trends of HCC over the last 10 years focusing on the aetiopathology of cirrhosis and modality of diagnosis. Hepatoma surveillance clinics have been established within the last 10 years, wherein cirrhotic patients are screened 6 monthly with ultrasound and alpha fetoprotein. We also aimed to compare the clinical characteristics of patients identified by this surveillance programme with those diagnosed when they presented with symptoms.

Methods Symptomatic HCC cases were identified by review of medical records coding with "Hepatocellular carcinoma" and the HPB multidisciplinary team meeting lists between 1/1/04 and 31/ $12 / 13$. The hepatoma screening programme records were accessed to identify cases detected on routine screening. Electronic patient records were used to identify the underlying pathology, date of diagnosis, date of death and other baseline data.

Results During this ten year period 146 individuals were diagnosed with HCC of which 25 cases were detected by the surveillance programme. The mean incidence of HCC rose from 11.6/ year during the period 2003-08 to 17.6/year in the years 200913. NAFLD was the most prevalent pathology in all years, but showed no significant change in incidence between these two time periods ( 42.1 vs. $36.6 \%)$. The mean age at diagnosis in the screened group was significantly lower $(57.1 \pm 8.3)$ than symptomatic $(69 \pm 9.7 ; \mathrm{p}<0.001)$. The rate of curative therapy (Resection, OLT) was higher in the screening group as opposed to the symptomatic (24 vs. $7.2 \%$ ) as also palliative therapy (TACE, RFA) (20 vs. 13.5\%). Transplant free survival was greater at 1 and 3 years in the screened group (35 vs. 25.5\%; 20 vs. $8.2 \%)$, but no significant difference was noted at 5 years $(0$ vs. $2 \%)$.

Conclusion We demonstrated a significant increase in incidence of HCC within our population in the last five years in keeping with other published studies, ${ }^{2}$ but no increase in NAFLD-related hepatoma. Within our population we have been able to show a positive effect of the hepatoma screening programme in terms of early age of diagnosis, suitability for a therapeutic option and better short and medium term survival rates.

\section{REFERENCES}

1 Fattovich G, Stroffolini T, Zagni I, Donato F. Hepatocellular carcinoma in cirrhosis: incidence and risk factors. Gastroenterology 2004;127(1):S35-S50

2 DavilaJA, El-Serag HB. The rising incidence of hepatocellular carcinoma in the United States: an update. Gastroenterology 2012;142(1):S914

Disclosure of Interest None Declared.

\section{PWE-137 THE BSG HEPATOLOGY DATASET: DOES IT WORK IN PRACTICE?}

${ }^{1} \mathrm{G}$ Sheiybani*, ${ }^{1} \mathrm{D}$ Durrant, ${ }^{2} \mathrm{~K}$ Pritchard, ${ }^{1} \mathrm{M}$ Bunting, ${ }^{1} \mathrm{JL}$ Brown, ${ }^{1} \mathrm{C}$ Hollywood. ${ }^{1}$ Gastroenterology, Gloucestershire Hospitals NHS Trust, Gloucester; ${ }^{2}$ Gastroenterology, Gloucestershire Hospitals NHS Trust, Bristol, UK

\subsection{6/gutjnl-2014-307263.397}

Introduction Clinical diagnosis and procedure coding for hospital admissions is mandatory in order to receive payment through the NHS tariff system. This does not extend into outpatient attendances where, to date, there has not been a nationally agreed process for coding. In order to address this, Connecting for Health (superseded by the Health and Social Care Information Centre), in 2013, invited clinical subspecialty societies to submit codes (limited to approximately 100) that would encapsulate $90 \%$ of their outpatient diagnoses. In this study we sought to validate the BSG hepatology subset* to see if it a) met the scope criteria for HSCIC and b) could be clinically useful in outpatient resource planning.

Methods We have studied the outpatient clinic letters in patients seen by hepatologists or hepatology nurse specialists in the 